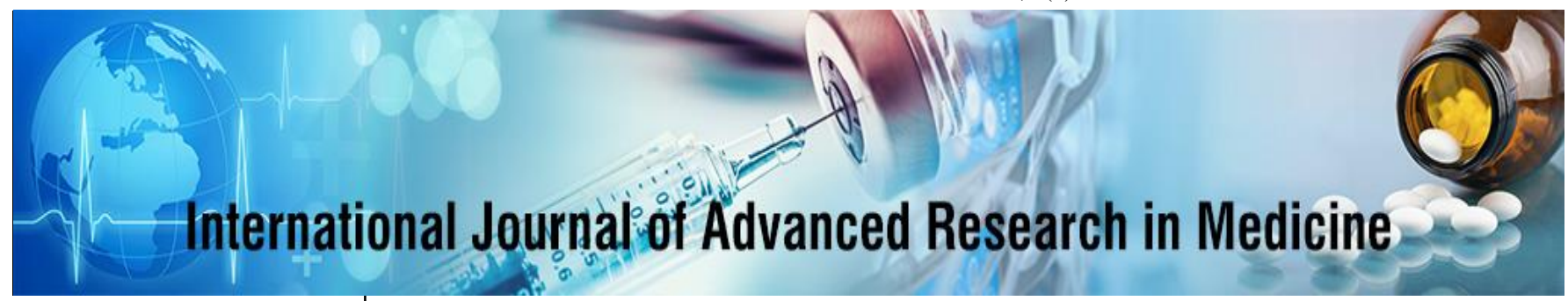

E-ISSN: 2706-9575

P-ISSN: 2706-9567

IJARM 2021; 3(1): 197-199

Received: 12-05-2020

Accepted: 19-06-2020

Dr. Manish Kumbhare

MD Medicine, Metro Hospital and Cancer Research Center,

Jabalpur, Madhya Pradesh, India

\section{Assessment of cases of ascites: A clinical study}

\author{
Dr. Manish Kumbhare
}

DOI: https://doi.org/10.22271/27069567.2021.v3.i1d.136

\begin{abstract}
Background: Ascites formation is the result of a series of anatomical, pathophysiological, and biochemical changes. The present study was conducted to assess cases of ascites in adult patients.

Materials and methods: 107 patients of ascites of both genders were involved. Physical findings and risk factors were recorded.

Results: Out of 107 patients, males were 67 and females were 40. Risk factors in patients were diabetes in 62, alcohol ingestion in 48 and blood transfusion in 54. The difference was non-significant $(\mathrm{P}>0.05)$. physical findings were murmur in 32 , elevated JVP in 20 , axillary hair loss in 18 , gynecomastia in 31 , icterus in 48 , palpable liver in 25 , pleural effusion in 39 and peripheral odema in 28 patients. The difference was significant $(P<0.05)$.

Conclusion: Common physical findings were murmur, elevated JVP, axillary hair loss, gynecomastia, icterus, palpable liver, pleural effusion and peripheral odema.
\end{abstract}

Keywords: Ascites, diabetes, palpable liver

\section{Introduction}

Ascites refers to the detectable and pathologic collection of fluid in the peritoneal cavity. Usually, it is a clinical finding and can be confirmed by a diagnostic paracentesis. Subclinical amount of fluid (i.e., less than 1.5 litre) can be detected using ultrasonography or computed tomography of the abdomen ${ }^{[1]}$.

Ascites formation is the result of a series of anatomical, pathophysiological, and biochemical changes. The specific causes of ascites can be divided into those associated with portal hypertension (cirrhotic ascites) and those unrelated to portal hypertension (non-cirrhotic ascites) ${ }^{[2]}$. In patients with liver cirrhosis, ascites develops as a consequence of sinusoidal portal hypertension, which results in alterations to capillary pressure, permeability and accumulation of retained fluid in the abdominal cavity. This mechanism of fluid accumulation is known as transudation. The passage of high molecular weight substances is limited because capillary damage is not the underlying process in transudation. Another mechanism of ascites formation is known as exudation; ascites development is secondary to increased vascular permeability due to the inflammatory process, tumoral invasion, or traumatic damage to the peritoneum or intraperitoneal organs ${ }^{[3]}$.

In a large number of patients, cirrhosis of liver is the cause of ascites. Several factors contribute to the development of ascites in chronic liver disease. Kidney plays a central role and is responsible for sodium and water retention, through complex mechanisms ${ }^{[4]}$. The mechanism by which the diseased liver affects renal function is not fully understood. The 'peripheral arterial vasodilatation hypothesis is based on the presence of characteristic circulatory abnormalities seen in cirrhotic patients. These patients show manifestations of increased cardiac output, arterial hypotension, decreased peripheral vascular resistance and splanchnic vasodilatation ${ }^{[5]}$. The present study was conducted to assess cases of ascites in adult patients.

\section{Materials and Methods}

The present study was conducted among 107 patients of ascites of both genders. All were informed regarding the study and written consent was obtained.

General information such as name, age, sex etc. was recorded. A general physical examination was performed in all patients. Parameters such as icterus, parotid swelling, and signs of ascites and investigations such as abdominal ultrasound were performed. 
Ascitic fluid was analyzed for biochemistry, cytology, gram staining, acid fast bacillus staining, malignant cells, culture and sensitivity. Serum-ascites albumin gradient (SAAG) and adenosine deaminase (ADA). Results thus obtained were subjected to statistical analysis. $\mathrm{P}$ value less than 0.05 was considered significant.

\section{Results}

Table 1: Distribution of patients

\begin{tabular}{|c|c|c|}
\hline \multicolumn{3}{|c|}{ Total- 107 } \\
\hline Gender & Males & Females \\
\hline Number & 67 & 40 \\
\hline
\end{tabular}

Table 1 shows that out of 107 patients, males were 67 and females were 40.

Table 2: Assessment of risk factors of ascites

\begin{tabular}{|c|c|c|}
\hline Risk factors & Number & P value \\
\hline Diabetes & 62 & \multirow{2}{*}{0.15} \\
\hline Alcohol ingestion & 48 & \\
\hline Blood transfusion & 54 & \\
\hline
\end{tabular}

Table 2, graph 1 shows that risk factors in patients were diabetes in 62, alcohol ingestion in 48 and blood transfusion in 54. The difference was non-significant $(\mathrm{P}>0.05)$.

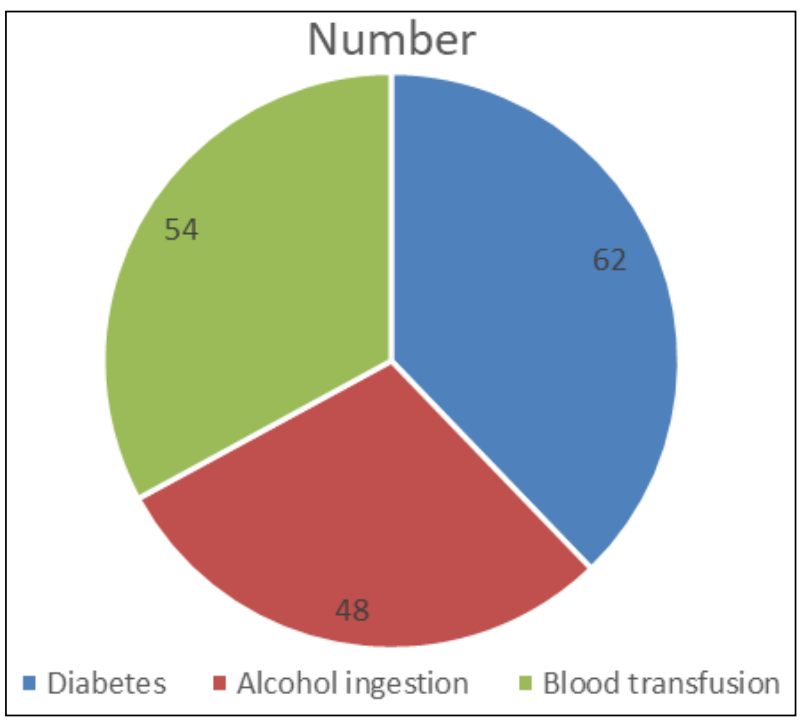

Graph 1: Assessment of risk factors of ascites

Table 3: Evaluation of physical findings

\begin{tabular}{|c|c|c|}
\hline Physical findings & Number & \multirow{2}{*}{ P value } \\
\hline Murmur & 32 & \\
\hline Elevated JVP & 20 & \multirow{2}{*}{0.01} \\
\hline Axillary hair loss & 18 & \\
\hline Gynecomastia & 31 & \\
\hline Icterus & 48 & \\
\hline Palpable liver & 25 & \\
\hline Pleural effusion & 39 & \\
\hline Peripheral odema & 28 & \\
\hline
\end{tabular}

Table 3, graph 2 shows that physical findings were murmur in 32, elevated JVP in 20, axillary hair loss in 18, gynecomastia in 31 , icterus in 48 , palpable liver in 25 , pleural effusion in 39 and peripheral odema in 28 patients. The difference was significant $(P<0.05)$.

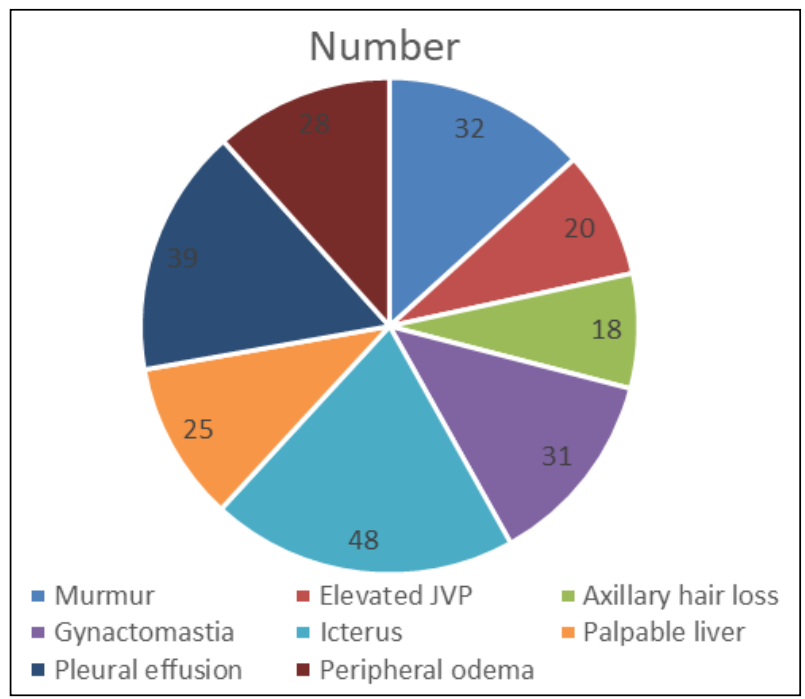

Graph 2: Evaluation of physical findings

\section{Discussion}

Ascitic fluid may accumulate rapidly or gradually depending upon the cause. Mild ascites may not produce any symptoms. Moderate ascites may just produce an increase in abdominal girth and weight gain. Large amounts of fluid can produce abdominal discomfort and the appearance of hernias particularly umbilical hernia and hinder the mobility of the patient. Ascites can be the first sign of liver disease. Thus, it is important to obtain a history of risk factors for a liver disease like alcohol consumption, drug abuse, blood transfusions, or hepatitis in the past ${ }^{[6]}$. The sudden development of ascites in a previously stable patient of cirrhosis should raise the suspicion of hepatoma. The differential diagnosis of ascites remains a problem in clinical practice. Treatment decisions are directed according to the etiological profile. Possible causes for vasodilatation in ascites include portosystemic shunting and/or impaired clearance of vasodilator substances like nitric oxide, endotoxins, prostacyclin, glucagon and adenosine ${ }^{[7]}$. This peripheral and splanchnic vasodilatation is perceived as reduction in effective plasma volume. The effective hypovolemia brings into play the baroreceptor mediated activation of renin-angiotensin-aldosterone system and sympathetic nervous system which produce renal vasoconstriction and salt ${ }^{[8]}$. The present study was conducted to assess cases of ascites in adult patients.

In present study, out of 107 patients, males were 67 and females were 40 . Patel et al. ${ }^{[9]}$ in their study on 150 patients of ascites found that cirrhosis of liver was the leading cause of ascites in $58.9 \%$, tuberculosis was the second most common cause of ascites (14.1\%), malignancy and cardiac disorders were the third $(15.3 \%)$ most common causes for ascites. Alcohol was the leading cause of cirrhosis in 64 patients $(72.7 \%)$.

We found that risk factors in patients were diabetes in 62 , alcohol ingestion in 48 and blood transfusion in 54. The initial evaluation of the gross appearance of ascitic fluid can offer useful information in the differential diagnosis. Under normal conditions, peritoneal fluid is clear to pale yellow [10]. Milky ascites, also called chylous ascites, is characterized by the presence of chylomicrons, which are lipoprotein particles that consist of large amounts of triglycerides. There are many known causes of chylous ascites, including cirrhosis, infections (parasitic and 
tuberculosis), malignancy, congenital defects, traumatism, inflammatory processes, nephropathies and cardiopathies. Abdominal malignancy is a major cause of chylous ascites in adults, whereas congenital lymphatic abnormalities are more likely causes in children [11]. However, it should be noted that pseudochylous ascites or cloudy/turbid ascites is associated with bacterial infection, peritonitis, pancreatitis, or perforated bowel. Therefore, the presence of both chylomicrons and a high concentration of triglycerides is necessary to distinguish chylous ascites from pseudochylous ascites. This is important since the frequency of malignancy is as high as $80 \%$ in adults with chylous ascites $^{[12]}$.

We observed that physical findings were murmur in 32 , elevated JVP in 20, axillary hair loss in 18, gynecomastia in 31 , icterus in 48 , palpable liver in 25 , pleural effusion in 39 and peripheral odema in 28 patients. According to MansourGhanaei et al. ${ }^{[13]}$ ascitic glucose concentration is often significantly lower than normal in tuberculous ascites, which makes it an indicator in differentiating tuberculosis from other diseases, such as cirrhosis. This is consistent with Wilkins et al. ${ }^{[14]}$ who recommended that the ascitic/blood glucose ratio is a useful test in the differentiation of tuberculous peritonitis from ascites due to other causes. However, when considering the value of glucose in patients with SAAG greater or less than $1.1 \mathrm{~g} / \mathrm{dL}$, there was no significant difference between them ${ }^{[15]}$. Therefore, due to its low diagnostic sensitivity and specificity, the application of ascitic glucose analysis is limited in routine practice.

\section{Conclusion}

Authors found that common physical findings were murmur, elevated JVP, axillary hair loss, gynecomastia, icterus, palpable liver, pleural effusion and peripheral odema.

\section{References}

1. Bataller R, Arroyo V, Gines P. Management of ascites in cirrhosis. J of Gastroenterol \& Hepatology 1997;12:72333.

2. Jalan R, Hayes PC. Hepatic encephalopathy and ascites. Lancet 1997;350:1309-15.

3. Schrier RW. Pathogenesis of sodium and water retention in high-output and low-output cardiac failure, nephrotic syndrome, cirrhosis, and pregnancy. N Engl J Med 1988;319:1127-34.

4. Runyon BA, Hoefs JC, Morgan TR. Ascitic fluid analysis in malignancy related ascites. Hepatology 1988;8:1104-09.

5. Press OW, Press NO, Kaufman SD. Evaluation and management of chylous ascites. Ann Intern Med 1982;96:358-64.

6. Cattau EL Jr, Benjamin SB, Knuff TE, Castell DO. The accuracy of the physical examination in the diagnosis of suspected ascites. JAMA 1982;247:1164-66.

7. Chongtham DS, Singh MM, Kalantri SP, Pathak S. A simple bedside manoeuvre to detect ascites. Natl Med J Ind 1997;10:13-4.

8. Runyon BA. Paracentesis of ascitic fluid: a safe procedure. Arch Intern Med 1986;146:2259-61.

9. Patel AB. A Study of 150 Cases of Ascites: Etiology and Diagnostic Evaluation. International Journal of Scientific Engineering and Research (IJSER) 2020;8:6-9.
10. Runyon BA. Care of patients with ascites. N Engl J Med 1994;330:337-42.

11. Bala L, Sharma A, Yellapa RK, Roy R, Choudhuri G, Khetrapal CL. 1H NMR spectroscopy of ascitic fluid: discrimination between malignant and benign ascites and comparison of the results with conventional methods. NMR Biomed 2008;21:606-614.

12. Lee HH, Carlson RW, Bull DM. Early diagnosis of spontaneous bacterial peritonitis: value of ascitic fluid variables. Infection 1987;15:232-236.

13. Mansour-Ghanaei F, Shafaghi A, Bagherzadeh AH, Fallah MS. Low gradient ascites: a seven year course review. World J Gastroenterol 2005;11:2337-2339.

14. Wilkins EG. Tuberculosis peritonitis: diagnostic value of the ascitic/blood glucose ratio. Tubercle 1984;65:47-52. 